\title{
Is awake the answer in high risk patients needing shoulder surgery?
}

\author{
AUTHORS: Gorecha M, Sainsbury KA, Dasgupta K and Bryant M \\ INSTITUION: George Eliot Hospital, Anaesthetic Dept, Nuneaton, UK
}

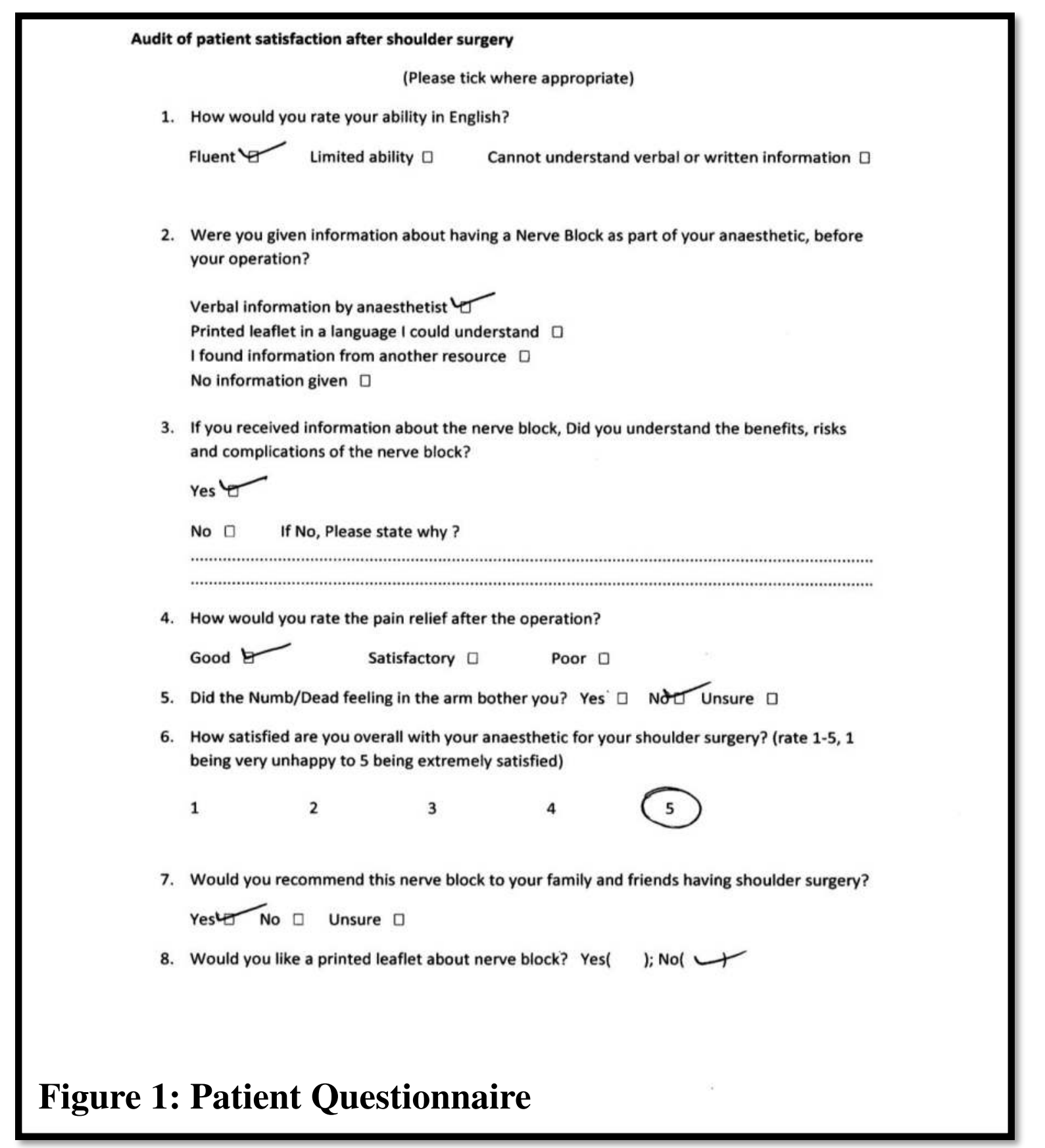

\section{BACKGROUND}

Shoulder surgery often requires the patient to be in the sitting position (beach chair) and this can cause dramatic haemodynamic instability.[1][2] General anaesthesia in elderly high-risk patients undergoing shoulder surgery in the sitting position can be potentially disastrous. Therefore, regional anaesthesia with sedation offers better haemodynamic stability. The majority of the shoulder is innervated by suprascapular and axillary nerves but the addition of superficial cervical plexus will prevent brachial plexus sparing which can occur in some operations if the incision is to medial from the shoulder.

\section{CASE REPORT}

An 80 year old man was scheduled for a right shoulder replacement. He had multiple medical problems including a previous stroke, drug eluting stent inserted into his left anterior descending coronary artery and still suffered from occasional angina for which he used a glyceryl trinitrate (GTN) spray. He was very short of breath on climbing one flight of stairs (a Duke activity status index of less than 4 METs). His medication history included bisoprolol, perindopril, aspirin, atorvastatin and warfarin which was stopped for one week preoperatively. A chest X-ray showed clear lung fields with some cardiomegaly. An echocardiogram showed severe LV impairment with an ejection fraction of 35\% by Simpson's method.[3] he was deemed very high risk for a general anaesthetic and a regional anaesthetic with sedation was planned. Preoperatively an arterial line was inserted so that blood pressure could be accurately controlled in the presence of atrial fibrillation. Sedation was started using a target controlled infusion (TCI) of remifentanil and propofol.[4] An ultrasound guided interscalene with a superficial cervical plexus block was used with $1 \%$ prilocaine $10 \mathrm{mls}$ and $0.375 \%$ levobupivacaine $20 \mathrm{mls}$ administered. We used an s-nerve sonsonite ${ }^{\circledR}$ ultrasound machine with a locoplex $50 \mathrm{~mm}$ vygon ${ }^{\circledR}$ nerve block needle. Intraoperatively the patient maintained haemodynamic stability and the surgery was successful. Within fifteen minutes of arrival in recovery he was able to have a cup of tea and was oriented in time and place . The patient was extremely satisfied with the outcome and completed a patient satisfaction survey (figure 1). He was admitted as an inpatient, his anticoagulation was managed by an anticoagulation specialist nurse He had good pain relief and was discharged from hospital after three days

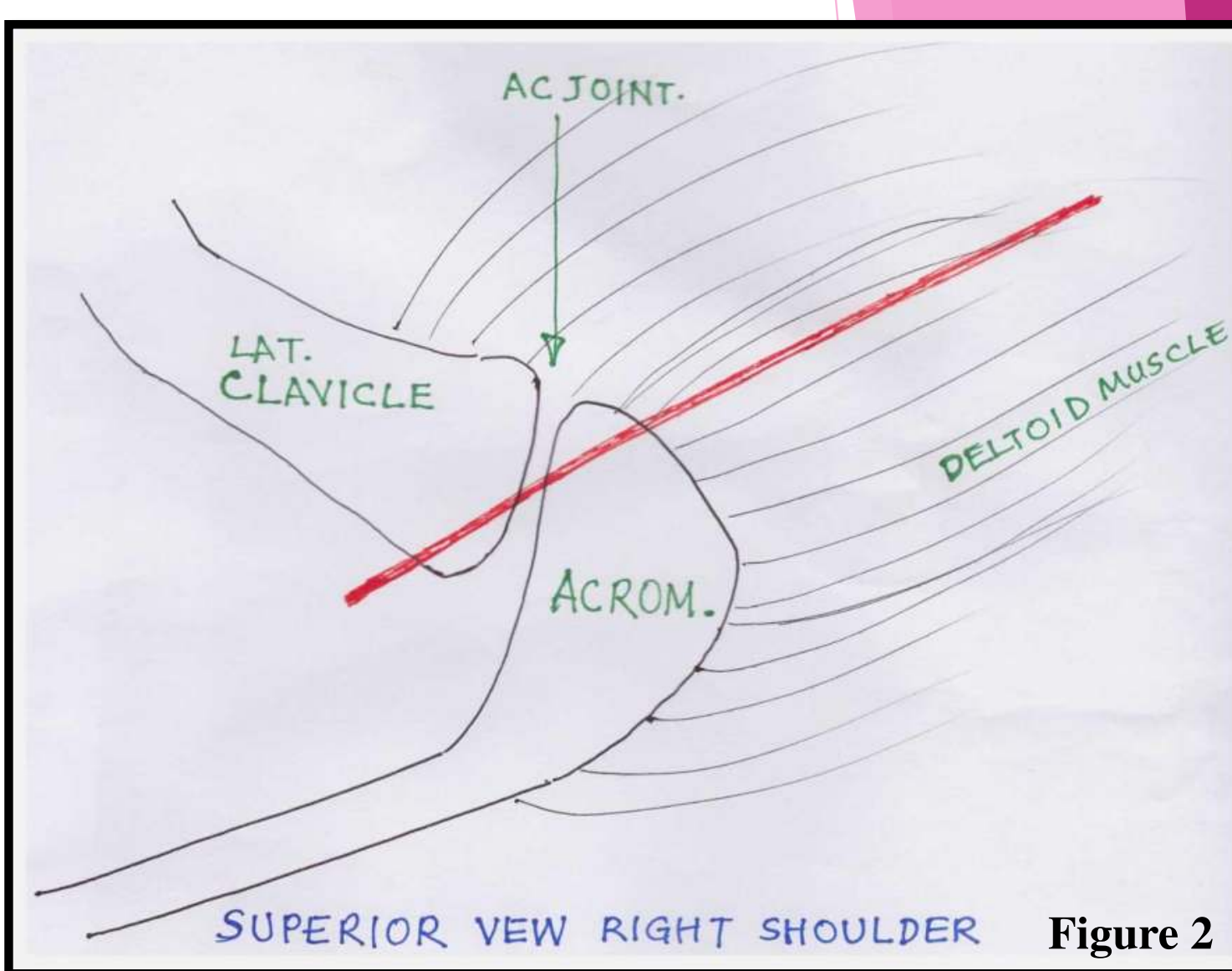

DISCUSSION

Sitting position (beach chair) is commonly used for shoulder surgery, but this position has advantages and disadvantages. Advantages are reduced brachial plexus strain, the shoulder remains in an anatomical position, better visualisation of the glenohumeral joint, and easier conversion to an open procedure. Disadvantages are embolic events, nerve damage and pressure points, irrigant solution complications, hypotension, and cerebral hypoperfusion.[5] Hypotension is due to venous pooling in the lower parts of the body and, in addition to the sympathetic block in the upper limb, causes a drastic decrease in venous return and can trigger the Bezold-Jarish reflex and hypotension. Because the patient had a previous history of stroke and MI, maintaining a mean arterial pressure of more than $80 \mathrm{mmHg}$ was our target, along with maintaining a heart rate of around $60-65 \mathrm{bpm}$. Tachycardia is very deleterious in a failing heart and is the most detrimental factor in managing Bezold-Jarisch reflex.[6] Keeping the patient awake during surgery also helped us to adequately assess cerebral perfusion considering his pervious history of stroke. In our hospital we do mostly posterior approach low interscalene blocks where the incidence of phrenic nerve palsy is very low and patients have no breathing difficulties. A superficial cervical plexus block was done to cover the superior part of the Mackenzie incision $(1 \mathrm{~cm}$ superomedial to the posterior edge of acromioclavicular joint), this is where brachial plexus block sparing occurs frequently (figure 2).[7]

\section{LEARNING POINTS}

- General anaesthesia in elderly patients present many problems

- Beach chair position shoulder surgery has many possible risks

- High risk patients undergoing shoulder surgery have the potential for high morbidity and mortality.

- Regional anaesthesia has tremendous advantages over general anaesthesia in this type of surgery by maintaining haemodynamic stability and cerebral and spinal cord perfusion, reducing post-operative confusion and allowing faster recovery and discharge.

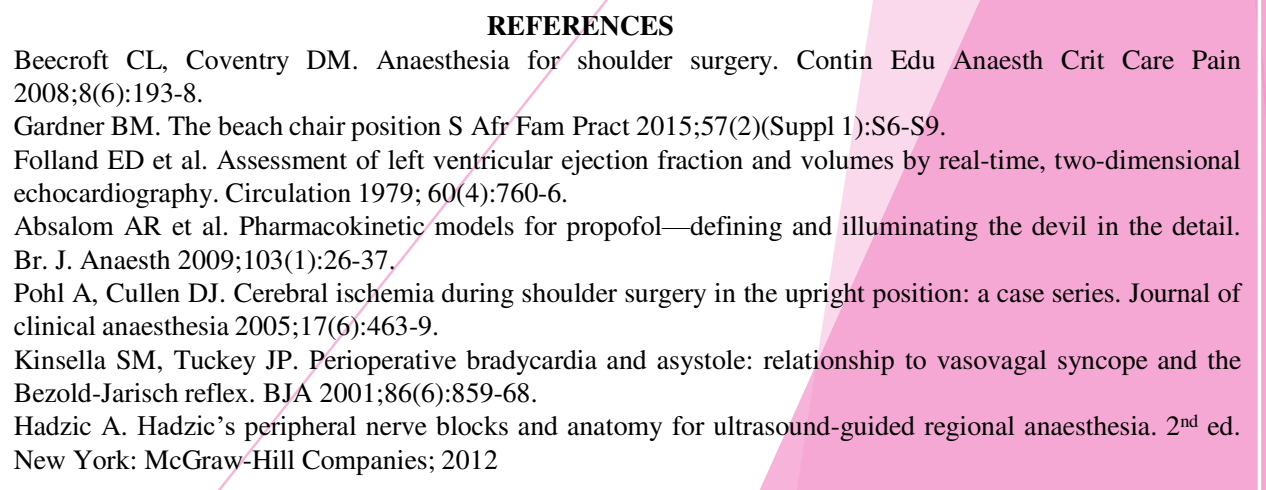

